\title{
Harninkontinenz: diskreter Schutz für den Mann
}

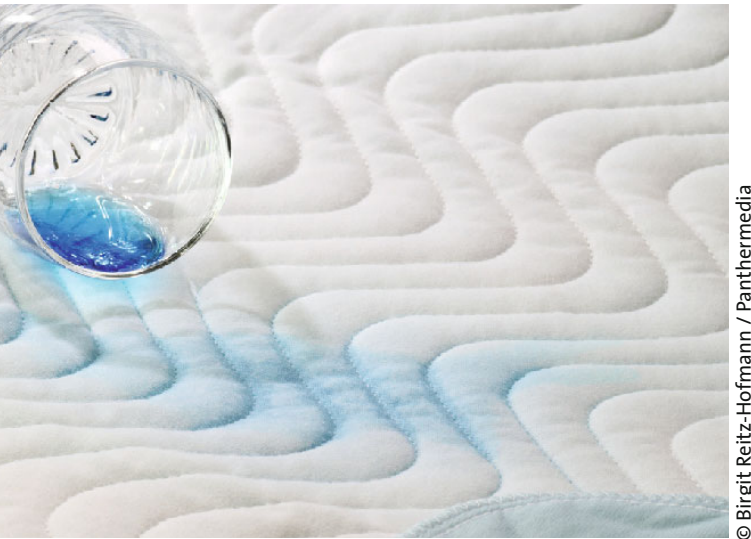

Eine Lösung bei Inkontinenz: Einlagen

— Blasenschwäche ist kein ausschließliches Frauenproblem, auch etwa jeder zehnte Vertreter des männlichen Geschlechts ist davon betroffen. Daher gibt es spezielle Inkontinenzprodukte für Männer, wie beispielsweise das neue TENA-Men-Sortiment.

Besonders Männer, die altersbedingt an einer Blasenschwäche leiden, sprechen nur ungern über ihr Problem und wissen daher oft nicht, dass es Inkontinenzprodukte für Männer gibt. Rund $77 \%$ der Betroffenen benutzen überhaupt keinen Schutz oder behelfen sich mit Taschentüchern.

Ein diskretes Einstiegsprodukt für Männer mit leichter Blasenschwäche ist „TENA Men Level 1". Die neue optimierte Version dieser Einlage ist kleiner und dünner als bisher. Dadurch können Männer sie unauffällig in der Unterwäsche tragen. Klebestreifen auf der Unterseite sorgen dafür, dass nichts verrutscht. Muss diese Einlage öfter als zweimal am Tag gewechselt werden, dann sollte eher die ebenfalls verbesserte, saugstärkere „TENA Men Level 2“ zum Einsatz kommen. Eine Besonderheit des TENAMen-Sortiments ist die Schalenform der Einlagen, die sich der männlichen Anatomie anpasst. Zudem sind die Einlagen mit dem Geruchsschutz „Odour Control ${ }^{\mathrm{TM}}$ “ versehen. Die TENA-Men-Rückseite ist atmungsaktiv, um die Hautverträglichkeit zu verbessern. Ebenfalls an die männliche Zielgruppe angepasst wurde die Verpackung, die auf der Vorderseite über die Saugstärke des Produkts informiert. Die Einlagen selbst sind einzeln verpackt und haben ein kleines Format, sodass sie überall hin diskret mitgenommen werden können.

Nach Informationen von

SCA Hygiene Products, Mannheim

\section{Neue Perspektive beim fortgeschrittenen Nierenzellkarzinom}

— Nach einer positiven Beurteilung des Committee for Medicinal Products for Human Use (CHMP) der Europäischen Arzneimittelagentur EMA wird die Zulassung von Pazopanib (Votrient ${ }^{\circledR}$ ) für die Behandlung des fortgeschrittenen Nierenzellkarzinoms ( $m R C C)$ nach vorangegangener Zytokinthe- rapie empfohlen. Prof. Hartmut Kirchner, Hannover, stellte die doppelblinde Phase-IIIStudie vor, die dem Zulassungsvotum zugrunde liegt [Sternberg et al. 2010]. $435 \mathrm{~Pa}$ tienten mit mRCC, die entweder nicht medikamentös vorbehandelt waren oder eine Erstlinientherapie mit Zytokinen erhalten

\section{Preis für Vardenafil wird erheblich gesenkt}

— Das Unternehmen Bayer Vital senkt zum 1. Mai 2010 den Apothekenverkaufspreis des Phosphodiesterase-5(PDE-5)Inhibitors Vardenafil (Levitra ${ }^{\circledR}$ ) für die Dosierungen $10 \mathrm{mg}$ und $5 \mathrm{mg}$ um etwa 50-60\%. Die Kosten pro 10 mg-Tablette sinken dabei von circa 12 EUR auf etwa 6 EUR. Ausschlag für diese Maßnahme gaben die Ergebnisse einer großen Marktstudie zum Einsatz von PDE-5-Inhibitoren. Die Studie zeigte, dass der Kostenfaktor für Patienten mit erektiler Dysfunktion entscheidend ist, da sie die Therapie meist selbst zahlen müssen. $50 \%$ der $\mathrm{Pa}$ tienten, die PDE-5-Hemmer einnehmen, teilen die Pillen. Dies ist aber problematisch, da so eine exakte Dosierung und eine kalkulierbare Resorption des Wirk- stoffs nicht gewährleistet sind. Zudem besorgen sich zahlreiche Patienten Pillen über das Internet. Wer Arzneimittel von unseriösen Anbietern erwirbt, läuft jedoch Gefahr, Fälschern aufzusitzen und seine Gesundheit zu gefährden.

„Vielen Männern mit Erektionsstörungen fällt es schon schwer, mit ihrem Problem überhaupt einen Arzt aufzusuchen“,fügt Dr. Herbert Schäfer von Bayer Vital hinzu. „Wenn sie es dann doch tun, wollen wir erreichen, dass sie nicht vor dem Preis zurückschrecken oder über dubiose Internetkäufe oder Tablettensplitting den Therapieerfolg gefährden."

Nach Informationen von Bayer Vital, Leverkusen hatten, wurden randomisiert entweder mit Pazopanib (einmal täglich $800 \mathrm{mg}, \mathrm{n}=290$ ) oder Placebo $(n=145)$ behandelt.

Im Pazopanib-Arm lag das mediane progressionsfreie Überleben (PFS) mit 9,2 Monaten signifikant höher als im Placeboarm (4,2 Monate). Nicht vorbehandelte Patienten profitierten noch deutlicher, berichtete Kirchner. Bei ihnen lag das PFS median bei 11,1 Monaten unter Pazopanib, im Placeboarm bei 2,8 Monaten. Aber auch mit Zytokin vorbehandelte Patienten hatten einen klar signifikanten Vorteil im PFS (7,4 vs. 4,2 Monate). Unerwünschte Ereignisse unter Pazopanib waren meist leicht bis mäßig ausgeprägt. Klasseneffekte für antiangiogenetisch wirkende Tyrosinkinase-Hemmer wie Proteinurie, Thrombozytopenie, Schilddrüsenunterfunktion, Hand-Fuß-Syndrom und Mukositis oder Stomatitis vom Schweregrad 3 oder 4 traten bei weniger als $1 \%$ der Patienten auf. Kirchner hält aufgrund des Nebenwirkungsspektrums von Pazopanib deutliche Vorteile gegenüber Sutinib für möglich.

fk

Satellitensymposium „Pazopanib - ein neuer Multityrosinkinase-Inhibitor in der klinischen Entwicklung bei soliden Tumoren" im Rahmen des 28. Deutschen Krebskongresses, Berlin. 26. Februar 2010 Veranstalter: GlaxoSmithKline, München 